Research Article

\title{
Risk Factors for Clinically Relevant Postoperative Pancreatic Fistula (CR-POPF) after Distal Pancreatectomy: A Single Center Retrospective Study
}

\author{
Gao Qing Wang $\mathbb{D}^{1},{ }^{1}$ Dipesh Kumar Yadav $\mathbb{D}^{2},{ }^{2}$ Wei Jiang ${ }^{\mathbb{D}},{ }^{1}$ Yong Fei Hua $\mathbb{D}^{1,2}$ \\ and Cai De Lu iD ${ }^{1}$ \\ ${ }^{1}$ Department of Hepatobiliary and Pancreatic Surgery, Ningbo Medical Center LiHuiLi Hospital, Ningbo 315040, \\ Zhejiang, China \\ ${ }^{2}$ Department of Hepatobiliary Surgery \& Liver Transplantation, The First Affiliated Hospital, Zhejiang University, \\ Hangzhou 310003, China
}

Correspondence should be addressed to Dipesh Kumar Yadav; 2338507593@qq.com and Cai De Lu; lucaide@nbu.edu.cn

Received 16 June 2020; Revised 15 November 2020; Accepted 7 January 2021; Published 20 January 2021

Academic Editor: Giovanni Marasco

Copyright (c) 2021 Gao Qing Wang et al. This is an open access article distributed under the Creative Commons Attribution License, which permits unrestricted use, distribution, and reproduction in any medium, provided the original work is properly cited.

\begin{abstract}
Objectives. Clinically relevant postoperative pancreatic fistula (CR-POPF) is the considerable contributor to major complications after pancreatectomy. The purpose of this study was to evaluate the potential risk factor contributing to CR-POPF following distal pancreatectomy (DP) and discuss the risk factors of pancreatic fistula in order to interpret the clinical importance. Methods. In this retrospective study, 263 patients who underwent DP at Ningbo Medical Center Li Huili Hospital between January 2011 and January 2020 were reviewed in accordance with relevant guidelines and regulations. Patients' demographics and clinical parameters were evaluated using univariate and multivariate analyses to identify the risk factors contributing to CR-POPF. $P<0.05$ was considered statistically significant. Results. In all of the 263 patients with DP, pancreatic fistula was the most common surgical complication (19.0\%). The univariate analysis of 18 factors showed that the patients with a malignant tumor, soft pancreas, and patient without ligation of the main pancreatic duct were more likely to develop pancreatic fistula. However, on multivariate analysis, the soft texture of the pancreas $(\mathrm{OR}=2.381,95 \% \mathrm{CI}=1.271-4.460, P=0.001)$ and the ligation of the main pancreatic $\operatorname{duct}(\mathrm{OR}=0.388,95 \% \mathrm{CI}=0.207-0.726, P=0.002)$ were only an independent influencing factor for CR-POPF. Conclusions. As a conclusion, pancreatic fistula was the most common surgical complication after DP. The soft texture of the pancreas and the absence of ligation of the main pancreatic duct can increase the risk of CR-POPF.
\end{abstract}

\section{Introduction}

In recent decades, distal pancreatectomy (DP) has become a common surgical technique for the treatment of benign and malignant pancreatic tumors, chronic pancreatitis, and pancreatic trauma [1]. Technically, DP is a simpler procedure compared to pancreaticoduodenectomy (PD), as a pancreatoenteric anastomosis is seldom required, and prevention of postoperative pancreatic fistula remains a challenge in DP due to an ineffective closure of the pancreatic remnant. The incidence of pancreatic fistula after DP ranges from 5\% to $32 \%$, depending upon the definition used and the underlying pancreatic pathology [2-6]. As per updated definition of the International Study Group for Pancreatic Fistula (ISGPF) in 2016, only grade B and grade C postoperative pancreatic fistulas are considered as a clinically relevant postoperative pancreatic fistula (CR-POPF) as it is associated with a clinically relevant development/condition related directly to the postoperative pancreatic fistula, and an earlier grade A postoperative pancreatic fistula is now no longer considered as a true pancreatic fistula because it has no clinical importance, instead it is now reported as a "biochemical leak" [7]. 
CR-POPF is the considerable contributor to major complications such as bleeding, abdominal abscess, sepsis, and even death following pancreatic resection [8-10]. Nevertheless, various attempts have been made to improve surgical outcomes that include suture closure of the pancreatic stump, staple transection of the pancreas, the use of fibrin glue to cover the pancreatic stump, coverage of the pancreatic stump with autologous tissue, the use of pancreatic stents, and the use of prophylactic octreotide [10]. Woefully, most of these methods have failed to improve fistula rates $[10,11]$. Nonetheless, risk identification and risk stratification might benefit in the prevention of POPF. Indeed, the development of the fistula risk score (FRS) for PD and its application has provided a great understanding for the prediction of POPF and has guided the modern accessible mitigation techniques in reduction of morbidity $[10,12]$. However, the underlying mechanism of POPF after DP is still poorly understood, and FRS for DP has not been developed yet that can predict the risk of POPF.

The purpose of this study was to evaluate the potential risk factors contributing to CR-POPF following DP and discuss the risk factors of pancreatic fistula to interpret the clinical importance.

\section{Patients and Methods}

2.1. Patients. All the patients who underwent DP at the Ningbo Medical Center Li Huili Hospital between January 2011 and January 2020 were reviewed retrospectively from the electronic medical record system. The study was approved by an Institutional Ethical Committee of the Ningbo Medical Center Li Huili Hospital and was consistent with the Declaration of Helsinki [13]. Written informed consent was obtained from all the patients or patients party before the surgery. The present data analysis includes 263 patients ( $n=213$ underwent open distal pancreatectomy and $n=50$ cases underwent laparoscopic distal pancreatectomy) undergoing DP over a 9-year period. Data were collected from the medical records on the standardized datasheets for all patients, and the variables collected were patients' demographics, surgery indications, preoperative evaluation and risk evaluation, preoperative lab values, perioperative and postoperative course that includes age, body mass index (BMI), smoking, and preoperative American Society of Anesthesiologists (ASA) risk grading [14], indication for surgery, pancreas texture, combined multivisceral resection, splenectomy, ligation of the main pancreatic duct, treatment of pancreatic stump, preoperative diabetes, intraoperative blood loss, use of somatostatin after surgery, preoperative albumin level, postoperative albumin level (3 days after surgery), surgical approach (open vs. laparoscopic), operation time, and pancreatic resection range.

2.2. Treatment Protocols. All patients underwent preoperative contrast-enhanced abdominal computed tomography or enhanced magnetic resonance imaging examination with cholangiopancreatography (MRCP) to accurately assess the nature of the lesion, location, size, and the relationship with the splenic vessels and other organs. Additionally, perioperative prophylactic antibiotics and a daily dose of low molecular weight heparin (LMWH) were given to all patients. Moreover, all of the patients also received prophylactic subcutaneous $200 \mu \mathrm{g}$ of octreotide as an induction dose. Nasogastric (NG) tubes were routinely placed throughout the operation. Furthermore, two tubes were generally placed at the end of an operation for drainage of fluid, i.e., a Jackson-Pratt drain (JP drain) near to the pancreatic stump remnant and another passive drainage tube in the operation field. Simultaneously, postoperative pain was managed by an epidural anesthesia or patientcontrolled analgesia (PCA), and all the patients were shifted to the intensive care unit (ICU) for a night. Besides, after surgery, some patients received a continuous intravenous infusion of octreotide at the rate of $0.25 \mathrm{mg} / \mathrm{hr}$ for 7 days with the help of a microinfusion pump on the random basis according to the surgeons preference.

Enhanced recovery after surgery (ERAS) protocol was used for postoperative management of all the patients, focusing on early mobilization and early nutrition intake [15]. Additionally, abdominal fluid drainage was monitored, and if the amount of drainage fluid was $<10 \mathrm{ml}$ after $24 \mathrm{hrs}$, the passive-drainage tube was withdrawn after an inspection with ultrasound to exclude any collection of fluid in the abdominal cavity. Moreover, the serum amylase level and drainage fluid amylase level (from JP drain) were examined after 3 days to rule out the presence of pancreatic fistula. In addition to this, at the time of follow-up, Doppler ultrasound was used see the patency of splenic vessels (for the patients with spleen-preserving DP with preservation of splenic vessels) and to rule out any thrombus or stricture in vessels. Furthermore, all the data were documented prospectively in the hospital database.

2.3. Operative Techniques. An operation was performed by 3 senior surgeons of our department. Moreover, the choice of surgical technique was decided by consultation between the surgeons and the patient party or according to underlying disease condition on preoperative radiological evaluation.

\subsubsection{Surgical Procedure for Laparoscopic DP.} Laparoscopic DP was mostly carried out for benign and low-grade malignant tumors in the distal pancreas. The surgical techniques for laparoscopic DP have already been described in detail in our previous studies [16, 17]. After ruling out any other abdominal pathology, metastasis, and any puncture to internal organs, abdominal surface of the pancreas was exposed by dissection of gastrocolic and gastrosplenic ligaments using a laparoscopic harmonic scalpel. Great care was taken to preserve the left gastroepiploic vessels and short gastric vessels. Furthermore, the dissection was performed according to the surgeons preference, and both superior-anterior approach [17] and inferior-posterior approach [16] are being used in our hospital for spleen-preserving DP with preservation of splenic vessels (Kimura technique [18]) by taking advantage of the avascular plain known as "the fusion fascia of Toldt" [16]. Warshaw technique 
[19] was only performed for low-grade malignant tumor with suspected or known cases of tumor invading the splenic vessels. After obtaining adequate surgical margin and after sufficient mobilization of the pancreas, the pancreas was divided proximally approximately $2 \mathrm{~cm}$ far from the tumor with the help of the Covidien Endo GIA Universal Straight $60-3.5 \mathrm{~mm}$ stapler. Additionally, in order to free the distal pancreatic stump together with the body and tail from the splenic vessels, it was dissected dorsally with the help of an ultrasonic knife by pulling it to the left lateral side. Furthermore, to minimise the risk of POPF, in the recent years, we routinely suture the pancreatic stump using polypropylene 3-0 intracorporeal interrupted sutures. Nonetheless, splenectomy was performed in case of an inadequate blood supply and outflow obstruction of the spleen. Additionally, taking oncologic principle into consideration, splenectomy was also performed if the tumor lies in close proximity to the splenic hilum $[20,21]$. At the end of the operation, the specimen was pulled out using a bag via an enlarged umbilical portsite incision and was sent for histopathology. Besides, the texture of the pancreas was determined by the tactile feedback of the instrument and was reassured after being pulled out from the abdominal cavity. Additionally, the texture of the pancreas was also confirmed from the histopathological report of the specimen after an operation based on the fibrosis grade of the pancreatic tissues. Last, the abdominal cavity was washed with warm water, and a JP drain tube was placed close to the pancreatic stump and a passive-drainage tube in the operation field on the left side through $5 \mathrm{~mm}$ port-site incisions.

\subsubsection{Surgical Procedure for Open DP. Open DP was carried} out both for benign tumor and malignant tumor in the distal pancreas. Open DP was performed with bilateral subcostal or upper midline incision. However, except the incision, other techniques were somewhat similar to laparoscopic DP. Nonetheless, in most of the cases, the transection of the pancreas was not performed with the Endo GIA stapler; the transection of pancreatic parenchyma was performed using the surgical blade, and the main pancreatic duct on the remnant pancreatic stump was ligated using 4-0 or 5-0 polypropylene continuous suture whenever identified. Additionally, the remnant pancreatic stump was also sutured using 4-0 or 5-0 polypropylene continuous suture to avoid any leakage from the branch pancreatic duct. In all the cases of malignant tumor, lymphadenectomy and the excision of the nodal tissues were performed along the common hepatic artery, the left gastric artery, the celiac axis, and along the superior mesenteric vein, including the peripancreatic lymph nodes. Additionally, extended resection along with resection of other visceral organs was performed in any cases of contiguous organ involvement.

2.4. Definitions. The severity grading of surgical complications was determined as proposed by Clavien-Dindo classification [22]. Moreover, the postoperative complications such as delayed gastric emptying (DGE), [23] postpancreatectomy hemorrhage (PPH) [24], chyle leak [25], and postoperative pancreatic fistula (POPF) [7] were in accordance with the consensus definition of the ISGPS. Precisely, a CR-POPF was defined as an external fistula with a drain output of any measurable volume of fluid after postoperative day 3 with an amylase level more than 3 times the upper limit, associated with a clinically relevant development/condition related directly to the POPF. Additionally, clinical criteria must be met in order to be considered as true POPF. Since, the earlier grade A POPF is not considered as true pancreatic fistula because it has no clinical importance; therefore, it was reported as a "biochemical leak." Only POPF grades B and C were placed in the category of CR-POPF. Particularly, grade B was defined as any changes required in the postoperative management; drains either left in place more than 3 weeks or repositioned through endoscopic or percutaneous procedures. Similarly, grade C POPF referred to those POPF that required reoperation or lead to single or multiple organ failure and/or mortality attributable to the pancreatic fistula [7]. Additionally, postoperative mortality was defined as the death within 30 days after surgery or death during the hospital stay [26].

2.5. Management of the Pancreatic Fistula. All the cases of POPF were managed by adequate tube drainage of the pancreatic stump, administration of octreotide, antibiotic therapy, irrigation, adjustment drainage of the tube in case of blockage, and gradual withdrawal of the drainage tube whenever the amylase and the lipase levels of the drain fluid were lower than 3 times the serum amylase level, and there was less than $50 \mathrm{ml}$ of fluid per day. Additionally, relaparotomy was performed for patients with grade C POPF. However, there was no standard treatment protocol for the management of POPF.

2.6. Statistical Analysis. All the statistical analyses were performed using SPSS 16.0 (IBM Corp., Armonk, NY). Continuous data are reported as a mean \pm standard deviation (SD). Categorical data are reported as absolute numbers $(n)$. The univariate analysis of risk factors for pancreatic fistula was performed by the $\chi^{2}$ test, and the multivariate analysis was performed by the multivariate logistic regression model (the backward elimination method) to test the independent risk factors for pancreatic fistula. $P<0.05$ was considered statistically significant.

\section{Results}

All 263 patients, including 124 males and 139 females, underwent DP at the Li Hui Li Hospital and the Ningbo medical center, Ningbo, between January 2011 and January 2020. The median age of the patients undergoing DP was 58 years (range 17-89 years). Of these 263 patients, 121 (46\%) had malignant tumors, and 142 (54\%) had benign or lowgrade malignant tumors (Table 1). Among total patients, 213 patients underwent open surgery, and 50 patients underwent laparoscopic surgery. The mean operation time was $221 \pm 90$ minutes (230 \pm 95 minutes for laparoscopic surgery and $219 \pm 83$ minutes for open surgery), and mean blood loss was 
TABLe 1: Primary lesions in 263 patients undergoing pancreatectomy.

\begin{tabular}{lcc}
\hline Primary lesion & Number of cases & Percentage (\%) \\
\hline Pancreatic cancer & 85 & 32.3 \\
Pancreatic solid-pseudopapillary tumor & 9 & 3.4 \\
Other organ malignancy (gastric cancer and lymphomas invading the pancreas) & 25 & 12 \\
Pancreatic trauma & 35 & 4.5 \\
Intraductal papillary mucinous neoplasm (IPMN) & 23 & 13.3 \\
Mucinous cystic neoplasm (MCN) & 22 & 8.7 \\
Pancreatic serous cystadenoma & 5 & 8.4 \\
Pancreatic cystadenocarcinoma & 4 & 1.9 \\
Primary pancreatic non-Hodgkin's lymphoma & 15 & 1.5 \\
Pancreatic pseudocyst & 2 & 5.7 \\
Pancreatic abscess & 12 & 0.8 \\
Pancreatic neuroendocrine tumors & 2 & 4.6 \\
Pancreatic neuroendocrine carcinoma & 1 & 0.8 \\
Intrapancreatic accessory spleen & 11 & 0.4 \\
Chronic pancreatitis & & 4.2 \\
\hline
\end{tabular}

$375 \pm 215 \mathrm{ml}$. There were 165 cases of combined splenectomy, whereas the spleen was preserved in 98 cases. However, endoscopy was not routinely performed at the time of follow-up; none of the patients suffered from gastric or esophageal variceal bleeding due to spleen-preserving DP. The mean follow-up time was $20.6 \pm 2.3$ months. Multivisceral resections were carried out in 74 (28.1\%) patients (4 patients had more than 2 combined organ resections) that include 32 partial gastrectomy, 8 adrenalectomies, 3 left nephrectomy, 18 partial hepatectomy, and 13 partial small intestine or colon resection (Table 2). Moreover, twenty-one patients had extended pancreatic body and tail resection (i.e., the pancreas was cut to the right side of the portal vein).

3.1. Management of Pancreatic Remnant. Mitigation techniques of the pancreatic remnant and resection margin were mainly performed by two techniques in our series, i.e., manual closure using sutures and closure using Endo GIA stapling. Manual closure using sutures was employed in 211 patients (80.2\%), whereas Endo GIA stapling was used in 52 patients $(19.7 \%)$. Of these, ligation of the main pancreatic duct was performed in 174 patients (66.1\%) overall. The incidence of CR-POPF was $23.1 \%$ in Endo GIA stapling and $18 \%$ in manual closure using sutures. However, the result was not statistically significant between the two.

3.2. Pancreatic Fistula and Other Complications. The total postoperative complications developed in 38.4\% (101/263) patients (i.e., one or more than one complication) that includes 50 cases of pancreatic fistula $(19.0 \%), 10$ cases of pulmonary infection (3.8\%), 5 cases of abdominal bleeding (1.9\%), 4 cases of cardiovascular complications $(1.5 \%), 4$ cases of chylous fistula (1.5\%), 1 case complicated with biliary fistula, gastric fistula, severe abdominal infection, and renal failure in a trauma patient, which was managed after active treatment $(0.4 \%)$, and 1 case of bile leakage in a patient with liver resection $(0.4 \%)$ (Table 3$)$. Among the patients who suffered from POPF, there were 61 cases of the biochemical leak, 48 cases (96.0\%) of grade B POPF, and 2 cases $(4.0 \%)$ of grade C POPF. The average time of hospital stay was 24.6 \pm 9.3 days in patients with POPF and $19.8 \pm 7.3$ days in patients without POPF $(P=0.025)$. Similarly, the average time of hospital stay was $20.5 \pm 7.5$ days in patients with biochemical leak and $31.5 \pm 9.2$ days in patients with CRPOPF (i.e., grade $\mathrm{B}$ and grade $\mathrm{C}$ POPF). The result was statistically significant between the two groups $(P=0.038)$. Postoperative complications due to CR-POPF occurred in 32 patients (64\%) include abdominal infection in 20 cases (40\%), delayed PPH in 2 cases (4\%), DGE in 5 cases (10\%), and surgical site wound infection in 5 cases (10\%). Fortunately, no postoperative mortality occurred in our series.

3.3. Risk Factors for the Development of CR-POPF. Furthermore, all 263 patients were divided into the CRPOPF group $(n=50)$ and non-CR-POPF group $(n=213)$ based on the occurrence of pancreatic fistula. The factors that might contribute in the development of pancreatic fistula are presented in Table 4. The univariate analysis of 18 factors showed that the patients with a malignant tumor, soft pancreas, and patients without ligation of the main pancreatic duct were more likely to develop pancreatic fistula. The incidence of CR-POPF in patients with malignant tumor was 30/121 (24.8\%) and 20/142 (14\%) in patients with benign disease or low-grade malignant tumors, $P=0.027$. Similarly, the incidence of CR-POPF in patients with the soft pancreas was $25 / 88(28.4 \%)$ and that of the firm pancreas was $25 / 175$ (14.2\%), $P=0.006$. Likewise, the incidence of CR-POPF in patients without ligation of the main pancreatic duct was $26 /$ $89(29.2 \%)$ and in patients with ligation of the main pancreatic duct was $24 / 174$ (13.8\%). $P=0.003$. However, univariate analysis demonstrated no significant relationship between CF-POPF and the following factors: age, BMI, smoking, ASA, combined multivisceral resection, splenectomy, pancreatic stump treatment, preoperative diabetes, intraoperative blood loss, use of somatostatin after surgery, preoperative albumin level, postoperative albumin level (3 days after surgery), surgical approach (open vs. laparoscopic), operation time, and pancreatic resection range. Only a significantly important association was demonstrated between CF-POPF and the following factors: pancreatic 
TABLe 2: Multivisceral distal pancreatectomy without the spleen $(n=74)$.

\begin{tabular}{lc}
\hline Organ (procedure) & Number \\
\hline Partial gastrectomy & 32 \\
Adrenalectomy & 8 \\
Left nephrectomy & 3 \\
Partial hepatectomy & 18 \\
Small intestine or colon resection & 13 \\
\hline
\end{tabular}

Note: 4 patients had more than 2 combined organ resections.

pathology (malignant tumor vs. benign disease or low-grade malignant tumor: $24.8 \%$ vs. $14 \%, P=0.027$ ), pancreas texture (soft vs. firm: $28.4 \%$ vs. $14.2 \%, P=0.006$ ), and ligation of the main pancreatic duct (no vs. yes: $29.2 \%$ vs. $13.8 \%, P=0.003)$.

Multivariate analysis was performed by the multivariate logistic regression model (the backward elimination method) for all 18 factors used in the univariate analysis. The results showed that the soft texture of the pancreas $(\mathrm{OR}=2.381,95 \% \mathrm{CI}=1.271-4.460, P=0.001)$ and the ligation of the main pancreatic duct $(\mathrm{OR}=0.388,95 \%$ $\mathrm{CI}=0.207-0.726, P=0.002)$ were independent influencing factors for CR-POPF (Table 5). The ligation of the main pancreatic duct was associated with lesser number of CRPOPF in the univariate analysis.

\section{Discussion}

In this study, we have examined both mortality and morbidity related to DP, with a particular aimed to POPF. Data from our study showed that DP can now be performed very safely without mortality. However, the higher rate of the morbidity still remains the concern, which was close to $38.4 \%$ in our series. Particularly, CR-POPF was the most frequent complication that occurred in $19 \%$ of our patients, and the rate of CR-POPF in our series is similar to that reported in the literature [27-29]. Nonetheless, CR-POPF is the considerable contributor to major complications such as peripancreatic effusion, peripancreatic abscess, pseudocyst formation, or erosion and digestion of surrounding tissues, resulting in intraabdominal hemorrhage and gastric emptying disorders, resulting in prolonged hospitalization time and increased hospitalization costs, affecting subsequent treatment following pancreatic resection [7-10]. Additionally, some patients may be readmitted after discharge due to the above complications. In our study, the length of hospital stay for the CR-POPF group was significantly longer than that of the non-CR-POPF group, with postoperative complications due to CR-POPF occurred in 32 patients (64\%) including bleeding.

On univariate analysis, CR-POPF occurred significantly at a higher rate in the soft pancreas (vs. the hard pancreas), and on the other hand, CR-POPF occurred significantly at a lower rate in the patients with benign disease or low-grade malignant tumor and when intraoperative ligation of the main pancreatic duct was not performed. No other factors were found to be related to an increased risk of CR-POPF.
However, on multivariate analysis, only the texture of the pancreas and the ligation of the main pancreatic duct were independent influencing factors for CR-POPF.

Many studies have revealed various preoperative, intraoperative, and postoperative variables as the risk factors for the development of CR-POPF, i.e., age, intraoperative blood loss, soft texture of the pancreas, BMI, multivisceral resections, splenectomy, operation time, gland thickness, and the fasting blood glucose level [27, 30-32]. However, most of these studies have been inconsistent with their findings with each other. The reasons for the inconsistent findings might be retrospective nature of the studies, heterogeneous practices among the surgeons, and the consequences of a learning curve for CR-POPF occurrence and management in different centers. Thus, the relationship between different risk factors for the development of CRPOPF should be interpreted cautiously. Nonetheless, in most of the studies, soft texture of the pancreas has widely been recognized as the most significant risk factor for the development of CR-POPF [12, 27, 32]. In our series, 88 patients had soft pancreatic texture (CR-POPF 28.4\%), and 175 patients had a hard pancreatic texture (CR-POPF 14.3\%). Indeed, univariate analysis revealed there were significant statistical differences for the development of CR-POPF between the two groups (soft pancreatic texture vs. hard pancreatic texture), $P=0.001$, attributing that the patients with soft pancreatic texture were more prone to develop a CR-POPF after DP than patients with a hard pancreatic texture. Additionally, multivariate analysis implied that a soft pancreatic texture was an independent risk factor associated with CR-POPF $(\mathrm{OR}=2.381,95 \% \mathrm{CI}=1.271-4.460$, $P=0.001)$. The lower rate of CR-POPF in patients with hard pancreatic texture may be explained by pancreatic fibrosis resulting into the exocrinal dysfunction of the pancreas. However, there are yet no standardized criteria to define the texture of the remnant pancreas as "soft" or "hard." In most of the cases, the texture of the remnant pancreas "soft" or "hard" is determined according to surgeon's own experience during the operation and confirmed by the histopathological report of the specimen after an operation based on the fibrosis grade of the pancreatic tissues. In recent years, some studies have proposed pancreatic elastography as an effective tool for the assessment of the pancreatic remnant texture and maybe used to predict POPF [33, 34]. Moreover, a recent meta-analysis found that a lower strain value on ultrasound shear wave elastography was significantly associated with CR-POPF. A lower strain value on ultrasound shear wave elastography implies to a softer pancreatic tissue. Thus, suggesting that the strain value on ultrasound elastography can be useful as an objective and quantifiable method to assess the pancreatic texture [35]. However, a recent prospective study by Marasco G. et al. concluded that pancreatic ultrasound elastography is not useful in predicting pancreatic fistula after pancreatic resection [36]. Nevertheless, the result of ultrasound elastography of the pancreas and the strain value could be influenced by various factors, such as the difference between ultrasound elastography equipment, the difference in ultrasound elastography techniques, steatosis of the pancreas, pulsations in the artery, 
TABle 3: Postoperative complications.

\begin{tabular}{lc}
\hline Complication & $N(\%)$ \\
\hline Pancreatic fistula & $50(19.0 \%)$ \\
Pulmonary infection & $10(3.8 \%)$ \\
Abdominal bleeding & $5(1.9 \%)$ \\
Cardiovascular complications & $4(1.5 \%)$ \\
Chylous fistula & $4(1.5 \%)$ \\
Biliary fistula, gastric fistula, severe abdominal infection, and renal failure in a trauma patient & $1(0.4 \%)$ \\
Bile leakage in a patient with liver resection & $1(0.4 \%)$ \\
\hline
\end{tabular}

obese patients, and the presence of gas in the bowel gas just in front of the pancreas [35]. Consequently, to address this controversy, well-designed prospective studies with a larger sample size are required for evaluating the accuracy of ultrasound elastography in the prediction of CR-POPF.

At present, the main mitigation strategies for the pancreatic remnant to reduce the risk of POPF include manual closure using sutures, closure using ENDO-GIA stapling, the use of fibrin glue to cover the pancreatic stump, coverage of the pancreatic stump with autologous tissue, the use of pancreatic stents, and the use of ultrasonic dissector [10,27]. Nonetheless, whether these mitigation strategies can reduce or prevent the occurrence of POPF is still debatable. There are several retrospective studies [27, 37], randomized controlled trials (RCTs) [4, 11, 38], and meta-analysis [39, 40] evaluating these mitigation strategies and found no evidence that these techniques are able to prevent or reduce risks of developing CR-POPF. Results from our study suggest that the intraoperative ligation of the main pancreatic duct can reduce the incidence of POPF; this observation was consistent with previous studies [41-43]. In our study, the incidence of CR-POPF was $29.2 \%$ when there was no intraoperative ligation of the main pancreatic duct and $13.8 \%$ when there was intraoperative ligation of the main pancreatic duct. Nevertheless, elective ligation of the main pancreatic duct might be difficult sometimes, especially when the main pancreatic duct is too thin, and it is difficult to be identified. To overcome such difficulties, we suggest sharp and careful transection of the pancreatic body or tail, where the main pancreatic duct can easily be identified in most of the cases. However, we should also acknowledge that only ligation of the main pancreatic duct is not an ultimate solution for POPF; the opening of the small branch ducts on the margin of the pancreatic remnant may also cause POPF. Because of the contractile resistance of the sphincter of Oddi, the pressure of the main pancreatic duct increases, which results in the formation of POPF due to the opening of the accessory branched pancreatic ducts on the pancreatic remnant. Thus, manual closure using sutures on the margin of the remnant pancreatic stump may be necessary. However, POPF can easily occur in the soft pancreas due to cutting and tearing of pancreatic tissue by sutures. Furthermore, if the suture is densely placed on the pancreatic remnant, it may cause ischemic necrosis of the tissue in the remnant pancreatic stump. Similarly, if the suture is placed too loose, it will cause POPF due to the incomplete suturing of the pancreatic stump. Thus, surgeons must take these factors into consideration while suturing the main pancreatic duct and the remnant pancreatic stump. However, some authors believe that the ligation of the main pancreatic duct does not affect the occurrence of pancreatic fistula $[27,29]$. It has been reported that preoperative endoscopic pancreatic stent implantation can effectively reduce the pressure of pancreatic exocrine ducts, thereby reducing the occurrence of pancreatic fistula [44]. Additionally, more recently, a study by Ecker et al. reported that the use of epidural analgesia was associated with significantly fewer incidences of POPF, probably because it is able to reduce the sphincter of Oddi pressure [27]. On the other hand, some authors believe that POPF can effectively be reduced by anastomosis of the pancreatic stump to the stomach (pancreaticogastrostomy) [45] or to the jejunum (pancreaticojejunostomy) [46] after DP [47]. However, the accuracy of these additional operations to prevent POPF remains to be further confirmed, but these additional surgical procedures undoubtedly will increase the complexity of the operation and prolong the time of the operation. In other words, this may increase the possibility of other postoperative complications. For the internal drainage of the pancreatic stump, the authors believe that, if preoperative imaging or intraoperative exploration reveals obstruction of the proximal pancreatic duct, the pancreatic stump should be anastomosed with the jejunum or the posterior gastric wall to drain the pancreatic juice, which may prevent POPF caused by the proximal pancreatic duct pressure.

Endo GIA stapling is a common method in DP for closure of the pancreatic stump, especially for laparoscopic surgery. It has advantages that it can save operation time and can be performed easily compared to the transection of pancreatic parenchyma using the surgical blade. However, there are some unfavourable factors, such as an inadequate ligation of the main pancreatic duct and tension at the edge of the pancreatic stump, which aggravate local ischemia and necrosis of the pancreatic stump. In our series, the incidence of CR-POPF in the Endo GIA stapling group was $23.1 \%$ and $18 \%$ in the suture group. However, there was no significant difference between both the groups. The reason we speculate for this is that, in the recent years, we routinely suture the pancreatic stump using polypropylene 3-0 intracorporeal interrupted sutures after Endo GIA stapling. Thus, this might have influenced the incidence of CR-POPF in the Endo GIA stapling group. Therefore, we believe that manual suture still remains the mainstream method for the treatment of the pancreatic stump after DP.

Our study has several limitations that need to be emphasized. First, this study is a retrospective nature and thus, 
TABLE 4: Univariate analysis of risk factors for postoperative pancreatic fistula after distal pancreatectomy (DP).

\begin{tabular}{|c|c|c|c|c|c|}
\hline Variables & $\begin{array}{c}\text { Number of cases, } \\
n=263\end{array}$ & $\begin{array}{l}\text { Pancreatic fistula, } \\
n=50\end{array}$ & $\begin{array}{c}\text { Nonpancreatic fistula, } \\
n=213\end{array}$ & $\chi^{2}$ & $\begin{array}{c}P \\
\text { value }\end{array}$ \\
\hline \multicolumn{6}{|l|}{ Age } \\
\hline$\geq 70$ & 45 & 9 & 36 & \multirow{2}{*}{0.034} & \multirow{2}{*}{0.853} \\
\hline$<70$ & 218 & 41 & 177 & & \\
\hline \multicolumn{6}{|l|}{ BMI $\left(\mathrm{kg} / \mathrm{m}^{2}\right)$} \\
\hline$>25$ & 84 & 11 & 73 & \multirow{2}{*}{2.806} & \multirow{2}{*}{0.094} \\
\hline$\leq 25$ & 179 & 39 & 140 & & \\
\hline \multicolumn{6}{|l|}{ Smoking } \\
\hline Yes & 67 & 13 & 54 & \multirow{2}{*}{0.009} & \multirow{2}{*}{0.925} \\
\hline No & 196 & 37 & 159 & & \\
\hline \multicolumn{6}{|l|}{ ASA } \\
\hline I & 114 & 21 & 93 & \multirow[b]{2}{*}{0.046} & \multirow[b]{2}{*}{0.831} \\
\hline II-III & 149 & 29 & 120 & & \\
\hline \multicolumn{6}{|l|}{ Indication for surgery } \\
\hline $\begin{array}{l}\text { Benign disease or low-grade malignant } \\
\text { tumors }\end{array}$ & 142 & 20 & 122 & \multirow[t]{2}{*}{4.866} & \multirow[t]{2}{*}{0.027} \\
\hline Malignant tumors & 121 & 30 & 91 & & \\
\hline \multicolumn{6}{|l|}{ Preoperative diabetes } \\
\hline Yes & 42 & 7 & 35 & \multirow{2}{*}{0.178} & \multirow{2}{*}{0.673} \\
\hline No & 221 & 43 & 178 & & \\
\hline \multicolumn{6}{|l|}{ Preoperative albumin level } \\
\hline$\geq 35 \mathrm{~g} / \mathrm{L}$ & 227 & 45 & 182 & 0711 & 0399 \\
\hline$<35 \mathrm{~g} / \mathrm{L}$ & 36 & 5 & 31 & 0.711 & 0.399 \\
\hline Surgical approach & & & & & \\
\hline Laparoscopic & 50 & 10 & 40 & & \\
\hline Open & 213 & 40 & 173 & 0.014 & 0.907 \\
\hline Operation time & & & & & \\
\hline$\geq 300 \mathrm{~min}$ & 54 & 10 & 44 & & \\
\hline$<300 \min$ & 209 & 40 & 169 & 0.011 & 0.918 \\
\hline Pancreas texture & & & & & \\
\hline Soft & 88 & 25 & 63 & & \\
\hline Hard & 175 & 25 & 150 & 7.586 & 0.006 \\
\hline Pancreatic resection range & & & & & \\
\hline Left side of portal vein & 242 & 45 & 197 & & \\
\hline Right side of portal vein & 21 & 5 & 16 & 0.341 & 0.559 \\
\hline Splenectomy & & & & & \\
\hline Yes & 165 & 29 & 136 & & \\
\hline No & 98 & 21 & 77 & 0.593 & 0.441 \\
\hline Combined multivisceral resection & & & & & \\
\hline Yes & 70 & 13 & 57 & & \\
\hline No & 193 & 37 & 156 & 0.012 & 0.913 \\
\hline Ligation of main pancreatic duct & & & & & \\
\hline Yes & 174 & 24 & 150 & & \\
\hline No & 89 & 26 & 63 & 9.094 & 0.003 \\
\hline Pancreatic stump treatment & & & & & \\
\hline Suture & 211 & 38 & 173 & & \\
\hline Endo GIA stapler & 52 & 12 & 40 & 0.696 & 0.404 \\
\hline Intraoperative blood loss & & & & & \\
\hline$\geq 600 \mathrm{ml}$ & 46 & 9 & 37 & & \\
\hline$<600 \mathrm{ml}$ & 217 & 41 & 176 & 0.269 & 0.604 \\
\hline Use of somatostatin after surgery & & & & & \\
\hline Yes & 75 & 14 & 61 & & \\
\hline No & 188 & 36 & 152 & 0.067 & 0.796 \\
\hline Postoperative albumin level (3 days after s & ery) & & & & \\
\hline$\geq 35 \mathrm{~g} / \mathrm{L}$ & 177 & 34 & 143 & & \\
\hline$<35 \mathrm{~g} / \mathrm{L}$ & 86 & 16 & 70 & 0.001 & 0.973 \\
\hline
\end{tabular}

BMI, body mass index. 
TABle 5: Multivariate logistic regression analysis for postoperative pancreatic fistula after distal pancreatectomy (DP).

\begin{tabular}{lcccccc}
\hline Variables & $\beta$ & S.E. & Wald & $P$ value & OR & $95 \%$ CI \\
\hline Pancreas texture (soft vs. hard) & -1.116 & 0.351 & 10.108 & 0.001 & 2.381 & $1.271-4.460$ \\
Ligation of the main pancreatic duct (yes vs. no) & -1.021 & 0.333 & 9.407 & 0.002 & 0.388 & $0.207-0.726$ \\
\hline
\end{tabular}

$\beta$, regression coefficient; S.E., standard error of regression coefficient; Wald, Wald chi-square value; CI, confidence interval; OR, odds ratio.

subject to biases. Likewise, the data included in this study are over a long period of time (2011-2020) and may have different surgical techniques and POPF mitigation strategies depending upon individual surgeon preference. Similarly, there might be a potential misgrading of patients with biochemical leakage before the updated definition of ISGPS 2016. Second, some clinical data are not sufficient like we could not collect proper data for pancreatic thickness, where different studies have outlined it as an independent risk factor for CR-POPF [48, 49]. Third, the effects of a learning curve on POPF occurrence and management of POPF cannot be excluded. Finally, our electronic medical record system might not have the record of complications that were managed in local hospitals. However, on the other hand, our study is still of great importance, as it includes large size of the cases from a single center. Moreover, we have analyzed most of the clinically relevant variables that might have an effect on the occurrence of POPF in both open and laparoscopic DP.

\section{Conclusion}

Pancreatic fistula was the most common surgical complication after DP. The soft texture of the pancreas and the absence of ligation of the main pancreatic duct can increase the risk of CR-POPF. No other factors such as age, BMI, smoking, ASA, combined multivisceral resection, splenectomy, pancreatic stump treatment, preoperative diabetes, intraoperative blood loss, use of somatostatin after surgery, preoperative albumin level, postoperative albumin level (3 days after surgery), surgical approach (open vs. laparoscopic), operation time, and pancreatic resection range had an influence on development of CR-POPF after DP.

\section{Data Availability}

The data used to support the findings of this study are included within the article and are available from the corresponding author upon request.

\section{Conflicts of Interest}

The authors declare that they have no conflicts of interest.

\section{Authors' Contributions}

Gao Qing Wang and Dipesh Kumar Yadav have equally contributed in study design, data collection, data analysis, and preparation of the manuscript. Gao Qing Wang, Dipesh Kumar Yadav, and Cai De Lu designed the study. Gao Qing Wang, Dipesh Kumar Yadav, Wei Jiang, and Yong Fei Hua collected data. Gao Qing Wang and Dipesh Kumar Yadav analyzed data and prepared the manuscript. Gao Qing
Wang, Dipesh Kumar Yadav, Wei Jiang, Yong Fei Hua, and Cai De Lu reviewed the final draft.

\section{Acknowledgments}

This study was funded by the Ningbo Natural Science Foundation of China (2019A610214) and Ningbo Health Branding Subject Fund (PPXK2018-03).

\section{References}

[1] H. Kawaida, H. Kono, M. Watanabe, N. Hosomura, H. Amemiya, and H. Fujii, "Risk factors of postoperative pancreatic fistula after distal pancreatectomy using a triplerow stapler," Surgery Today, vol. 48, no. 1, pp. 95-100, 2018.

[2] M. Sugimoto, N. Gotohda, Y. Kato et al., "Risk factor analysis and prevention of postoperative pancreatic fistula after distal pancreatectomy with stapler use," Journal of Hepato-BiliaryPancreatic Sciences, vol. 20, no. 5, pp. 538-544, 2013.

[3] K. Okano, M. Oshima, K. Kakinoki et al., "Pancreatic thickness as a predictive factor for postoperative pancreatic fistula after distal pancreatectomy using an endopath stapler," Surgery Today, vol. 43, no. 2, pp. 141-147, 2013.

[4] M. K. Diener, C. M. Seiler, I. Rossion et al., "Efficacy of stapler versus hand-sewn closure after distal pancreatectomy (DISPACT): a randomised, controlled multicentre trial," The Lancet, vol. 377, no. 9776, pp. 1514-1522, 2011.

[5] H. Eguchi, H. Nagano, M. Tanemura et al., "A thick pancreas is a risk factor for pancreatic fistula after a distal pancreatectomy: selection of the closure technique according to the thickness," Digestive Surgery, vol. 28, no. 1, pp. 50-56, 2011.

[6] H. Seeliger, S. Christians, M. K. Angele et al., "Risk factors for surgical complications in distal pancreatectomy," The American Journal of Surgery, vol. 200, no. 3, pp. 311-317, 2010.

[7] C. Bassi, G. Marchegiani, C. Dervenis et al., "The 2016 update of the International Study Group (ISGPS) definition and grading of postoperative pancreatic fistula: 11 Years after," Surgery, vol. 161, no. 3, pp. 584-591, 2017.

[8] M. K. Lee, R. S. Lewis, S. M. Strasberg et al., "Defining the post-operative morbidity index for distal pancreatectomy," Hpb Journal, vol. 16, no. 10, pp. 915-923, 2014.

[9] W. B. Pratt, S. K. Maithel, T. Vanounou, Z. S. Huang, M. P. Callery, and C. M. Vollmer, "Clinical and economic validation of the international study group of pancreatic fistula (ISGPF) classification scheme," Annals of Surgery, vol. 245, no. 3, pp. 443-451, 2007.

[10] C. Nahm, S. Connor, J. Samra, and A. Mittal, "Postoperative pancreatic fistula: a review of traditional and emerging concepts," Clinical and Experimental Gastroenterology, vol. 11, pp. 105-118, 2018.

[11] Y. Miyasaka, Y. Mori, K. Nakata, T. Ohtsuka, and M. Nakamura, "Attempts to prevent postoperative pancreatic fistula after distal pancreatectomy," Surgery Today, vol. 47, no. 4, pp. 416-424, 2017.

[12] M. P. Callery, W. B. Pratt, T. S. Kent, E. L. Chaikof, and C. M. Vollmer Jr., "A prospectively validated clinical risk 
score accurately predicts pancreatic fistula after pancreatoduodenectomy," Journal of the American College of Surgeons, vol. 216, no. 1, pp. 1-14, 2013.

[13] A. Y. Malik and C. Foster, "The revised Declaration of Helsinki: cosmetic or real change?" Journal of the Royal Society of Medicine, vol. 109, no. 5, pp. 184-189, 2016.

[14] D. J. Doyle and E. H. Garmon, "American society of Anesthesiologists classification (ASA class)," in StatPearlsStatPearls Publishing LLC., Treasure Island, FL, USA, 2019.

[15] G.-R. Joliat, I. Labgaa, D. Petermann et al., "Cost-benefit analysis of an enhanced recovery protocol for pancreaticoduodenectomy," British Journal of Surgery, vol. 102, no. 13, pp. 1676-1683, 2015.

[16] Y. F. Hua, D. K. Yadav, X. Bai, and T. Liang, "Laparoscopic spleen-preserving distal pancreatectomy (LSPDP) with preservation of splenic vessels: an inferior-posterior approach," Gastroenterology Research and Practice, vol. 2018, Article ID 1683719, 2018.

[17] J. Huang, D. K. Yadav, C. Xiong et al., "Laparoscopic spleenpreserving distal pancreatectomy (LSPDP) versus open spleen-preserving distal pancreatectomy (OSPDP): a comparative study," Canadian Journal of Gastroenterology and Hepatology, vol. 2019, p. 7, 2019.

[18] W. Kimura, M. Yano, S. Sugawara et al., "Spleen-preserving distal pancreatectomy with conservation of the splenic artery and vein: techniques and its significance," Journal of HepatoBiliary-Pancreatic Sciences, vol. 17, no. 6, pp. 813-823, 2010.

[19] A. L. Warshaw, "Conservation of the spleen with distal pancreatectomy," Archives of Surgery (Chicago, Ill: 1960, vol. 123, no. 5, pp. 550-553, 1988.

[20] S. M. Strasberg, J. A. Drebin, and D. Linehan, "Radical antegrade modular pancreatosplenectomy," Surgery, vol. 133, no. 5, pp. 521-527, 2003.

[21] J. Christein, M. Kendrick, C. Iqbal, D. Nagorney, and M. Farnell, "Distal pancreatectomy for resectable adenocarcinoma of the body and tail of the pancreas," Journal of Gastrointestinal Surgery, vol. 9, no. 7, pp. 922-927, 2005.

[22] P. A. Clavien, J. Barkun, M. L. De Oliveira et al., "The claviendindo classification of surgical complications," Annals of Surgery, vol. 250, no. 2, pp. 187-196, 2009.

[23] M. N. Wente, C. Bassi, C. Dervenis et al., "Delayed gastric emptying (DGE) after pancreatic surgery: a suggested definition by the International Study Group of Pancreatic Surgery (ISGPS)," Surgery, vol. 142, no. 5, pp. 761-768, 2007.

[24] M. N. Wente, J. A. Veit, C. Bassi et al., "Postpancreatectomy hemorrhage (PPH)-An international study group of pancreatic surgery (ISGPS) definition," Surgery, vol. 142, no. 1, pp. 20-25, 2007.

[25] M. G. Besselink, L. B. Van Rijssen, C. Bassi et al., "Definition and classification of chyle leak after pancreatic operation: a consensus statement by the International study group on pancreatic surgery," Surgery, vol. 161, no. 2, pp. 365-372, 2017.

[26] J. P. Jacobs, C. Mavroudis, M. L. Jacobs et al., "What is operative mortality? defining death in a surgical registry database: a report of the STS congenital database taskforce and the Joint EACTS-STS congenital database committee," The Annals of Thoracic Surgery, vol. 81, no. 5, pp. 1937-1941, 2006.

[27] B. L. Ecker, M. T. McMillan, V. Allegrini et al., "Risk factors and mitigation strategies for pancreatic fistula after distal pancreatectomy," Annals of Surgery, vol. 269, no. 1, pp. 143-149, 2019.

[28] S. Dokmak, F. S. Ftériche, R. L. Meniconi et al., "Pancreatic fistula following laparoscopic distal pancreatectomy is probably unrelated to the stapler size but to the drainage modality and significantly decreased with a small suction drain," Langenbeck's Archives of Surgery, vol. 404, no. 2, pp. 203-212, 2019.

[29] H. Nathan, J. L. Cameron, C. R. Goodwin et al., "Risk factors for pancreatic leak after distal pancreatectomy," Annals of Surgery, vol. 250, no. 2, pp. 277-281, 2009.

[30] J. R. Kleeff, M. K. Diener, K. Graggen et al., "Distal pancreatectomy," Annals of Surgery, vol. 245, no. 4, pp. 573-582, 2007.

[31] Z. Ke, J. Cui, N. Hu et al., "Risk factors for postoperative pancreatic fistula: analysis of 170 consecutive cases of pancreaticoduodenectomy based on the updated ISGPS classification and grading system," Medicine (Baltimore), vol. 97, no. 35, Article ID e12151, 2018.

[32] T. Xia, J. Y. Zhou, Y. P. Mou et al., "Risk factors for postoperative pancreatic fistula after laparoscopic distal pancreatectomy using stapler closure technique from one single surgeon," PLoS One, vol. 12, no. 2, Article ID e0172857, 2017.

[33] M. Hatano, J. Watanabe, F. Kushihata et al., "Quantification of pancreatic stiffness on intraoperative ultrasound elastography and evaluation of its relationship with postoperative pancreatic fistula," International Surgery, vol. 100, no. 3, pp. 497-502, 2015.

[34] M. Sugimoto, S. Takahashi, M. Kojima et al., "What is the nature of pancreatic consistency? assessment of the elastic modulus of the pancreas and comparison with tactile sensation, histology, and occurrence of postoperative pancreatic fistula after pancreaticoduodenectomy," Surgery, vol. 156, no. 5, pp. 1204-1211, 2014.

[35] G. Marasco, C. Ricci, F. Buttitta et al., "Is ultrasound elastography useful in predicting clinically relevant pancreatic fistula after pancreatic resection?" Pancreas, vol. 49, no. 10, pp. 1342-1347, 2020.

[36] G. Marasco, C. Ricci, V. Grasso et al., "Pancreatic ultrasound elastography is not useful to predict the risk of pancreatic fistulas after pancreatic resection," Updates in Surgery, vol. 72, no. $4,2020$.

[37] B. N. Fahy, C. F. Frey, H. S. Ho, L. Beckett, and R. J. Bold, "Morbidity, mortality, and technical factors of distal pancreatectomy," The American Journal of Surgery, vol. 183, no. 3, pp. 237-241, 2002.

[38] T. I. Carter, Z. V. Fong, T. Hyslop et al., "A dual-institution randomized controlled trial of remnant closure after distal pancreatectomy: does the addition of a falciform patch and fibrin glue improve outcomes?" Journal of Gastrointestinal Surgery, vol. 17, no. 1, pp. 102-109, 2013.

[39] P. Probst, F. J. Huttner, U. Klaiber et al., "Stapler versus scalpel resection followed by hand-sewn closure of the pancreatic remnant for distal pancreatectomy," The Cochrane Database of Systematic Reviews, vol. 11, Article ID Cd008688, 2015.

[40] H. P. Knaebel, M. K. Diener, M. N. Wente, M. W. Büchler, and C. M. Seiler, "Systematic review and meta-analysis of technique for closure of the pancreatic remnant after distal pancreatectomy," British Journal of Surgery, vol. 92, no. 5, pp. 539-546, 2005.

[41] M. M. Bilimoria, J. N. Cormier, Y. Mun, J. E. Lee, D. B. Evans, and P. W. T. Pisters, "Pancreatic leak after left pancreatectomy is reduced following main pancreatic duct ligation," British Journal of Surgery, vol. 90, no. 2, pp. 190-196, 2003.

[42] V. Pannegeon, P. Pessaux, A. Sauvanet, M. P. Vullierme, R. Kianmanesh, and J. Belghiti, "Pancreatic fistula after distal pancreatectomy: predictive risk factors and value of conservative treatment," Archives of Surgery (Chicago, Ill: 1960), vol. 141, no. 11, pp. 1071-1076, 2006. 
[43] M. Distler, S. Kersting, F. Rückert et al., "Chronic pancreatitis of the pancreatic remnant is an independent risk factor for pancreatic fistula after distal pancreatectomy," BMC Surgery, vol. 14, p. 54, 2014.

[44] N. Abe, M. Sugiyama, Y. Suzuki et al., "Preoperative endoscopic pancreatic stenting for prophylaxis of pancreatic fistula development after distal pancreatectomy," The American Journal of Surgery, vol. 191, no. 2, pp. 198-200, 2006.

[45] H. Yanagimoto, S. Satoi, H. Toyokawa et al., "Pancreaticogastrostomy following distal pancreatectomy prevents pancreatic fistula-related complications," Journal of HepatoBiliary-Pancreatic Sciences, vol. 21, no. 7, pp. 473-478, 2014.

[46] M. Kawai, S. Hirono, K.-I. Okada et al., "Randomized controlled trial of pancreaticojejunostomy versus stapler closure of the pancreatic stump during distal pancreatectomy to reduce pancreatic fistula," Annals of Surgery, vol. 264, no. 1, pp. 180-187, 2016.

[47] E. Tieftrunk, I. E. Demir, S. Schorn et al., "Pancreatic stump closure techniques and pancreatic fistula formation after distal pancreatectomy: meta-analysis and single-center experience," PLOS one, vol. 13, no. 6, Article ID e0197553, 2018.

[48] H. Kim, J.-Y. Jang, D. Son et al., "Optimal stapler cartridge selection according to the thickness of the pancreas in distal pancreatectomy," Medicine, vol. 95, no. 35, Article ID e4441, 2016.

[49] M. Kawai, M. Tani, K.-I. Okada et al., "Stump closure of a thick pancreas using stapler closure increases pancreatic fistula after distal pancreatectomy," The American Journal of Surgery, vol. 206, no. 3, pp. 352-359, 2013. 